\title{
SATISFACCIÓN CON LA VIDA Y CAPACIDADES FINANCIERAS EN ADULTOS MAYORES CON ENVEJECIMIENTO ACTIVO: ESTUDIO EXPLORATORIO EN EL SUR DE CHILE
}

\section{LIFE SATISFACTION AND FINANCIAL CAPABILITIES IN OLDER ADULTS WITH ACTIVE AGING: EXPLORATORY STUDY IN SOUTHER CHILE}

\author{
Regina M. Navarro-Blanco ${ }^{1}$ \\ Marianela Denegri-Coria ${ }^{2}$ \\ Verónica Peñaloza ${ }^{3}$
}

\begin{tabular}{|l|l} 
Recibido: 20.10 .19 & Aprobado: 30.04.20
\end{tabular}

DOI: $10.15517 /$ isucr.v21i43.41977

\section{Resumen}

El rápido envejecimiento de la población mundial, incentiva el interés por la investigación de los adultos mayores desde la perspectiva psicosocial, económica y del envejecimiento activo, considerado impactos y relaciones entre estos aspectos. En este escenario, Chile se posiciona como el tercer país con más rápido envejecimiento en América Latina. Por ello y desde la óptica de la psicología económica, el objetivo de este primer estudio fue explorar la satisfacción con la vida, actitudes hacia el endeudamiento, las capacidades financieras y posibles relaciones entre estas variables, en una muestra de adultos mayores con envejecimiento activo en el Sur de Chile. Se aplicó un cuestionario a 101 adultos mayores, incluyendo las escalas de Satisfacción con la Vida (Diener et al., 1985), de Actitudes hacia el Endeudamiento (Denegri, et al., 2012), y de Independencia y Seguridad Financiera (Carpenter \& Moore, 2008). Como parte de la exploración, se hizo análisis factorial exploratorio para cada una de las escalas. Los instrumentos presentaron adecuados niveles de consistencia interna. Se encontró correlación directa y positiva $(r=0,365, p$ $<0,001)$ entre la satisfacción con la vida y la actitud hacia el endeudamiento austero, así como con la autopercepción de independencia y seguridad en el manejo de las finanzas personales $(r=0,367$, $p<0,001)$, y entre esto último y el estilo de endeudamiento austero $(r=0,356, p<0,001)$. Se discuten los resultados y sus implicaciones.

Palabras clave: Satisfacción con la vida; capacidades financieras; actitudes hacia el endeudamiento; independencia y seguridad financiera; adultos mayores.

\footnotetext{
${ }^{1}$ Docente Departamento de Psicología y Centro de Excelencia en Psicología Económica, Universidad de La Frontera, Temuco, Chile. Email: regina.navarro@ufrontera.cl

${ }^{2}$ Docente Centro de Excelencia en Psicología Económica y del Consumo, Núcleo Científico y Tecnológico en Ciencias Sociales, Departamento de Psicología, Universidad de La Frontera, Temuco, Chile. Email: marianela.denegri@ufrontera.cl

${ }^{3}$ Docente Universidad de Ceará (Brasil), Centro de Excelencia en Psicología Económica, Universidad de la Frontera, Temuco, Chile. Email: veronica.penaloza@uece.br
} 


\begin{abstract}
The rapid aging of the world population, encourages an interest in the research of older adults from the perspective psychosocial, economic and of active aging, considering impact and relationship between this aspects. In this field, Chile is positioned as the third country with the fastest aging in Latin America. For this and from the perspective of economic psychology, the aim of this first study is to explore the life satisfaction, financial capabilities and possible relationships between these variables in a sample of older adults with active aging in Souther Chile. A questionnaire was applied to 101 older adults, which included the Satisfaction with Life scale (Diener et al., 1985), Attitudes toward Indebtedness (Denegri et al., 2012), and Security and Financial Independence (Carpenter \& Moore, 2008). As part of the exploration, exploratory factor analysis was done for each scale. The instruments presented adequate levels of internal consistency. A direct and positive correlation $(\mathrm{r}=0.365, \mathrm{p}<0.001)$ was found between life satisfaction and the attitude towards austere indebtedness, as well as self-perception of independence and security in the management of personal finances $(\mathrm{r}=0.367, \mathrm{p}<0.001)$, and between the austere indebtedness style $(\mathrm{r}=0.356$, $\mathrm{p}<0.001)$. These results and their implications are discussed.
\end{abstract}

Keywords: Satisfaction with life; financial capabilities; attitudes toward indebtedness; financial independence and security; older adult.

\title{
Introducción
}

En los últimos 50 años, debido a la disminución de las tasas de natalidad y a los avances en los conocimientos médicos y tecnológicos, la esperanza de vida al nacer ha aumentado. En este escenario, aproximadamente un millón de personas a nivel mundial llega a los 60 años todos los meses y el cambio demográfico será más rápido e intenso en los países de ingresos bajos y medianos (Organización Mundial de la Salud [OMS], 2015).

Chile ha mostrado una drástica disminución en sus tasas de natalidad, lo que tiene como resultado un envejecimiento en etapa avanzada y a ritmo sostenido (Instituto Nacional de Estadística [INE], 2017), siendo el tercer país con esta característica en América Latina y el Caribe, después de Cuba y Uruguay (Comisión Económica para América Latina y el Caribe [CEPAL], 2017; González, Massad, \& Lavanderos, 2009; Huenchuan, 2013). La IX región del país (en la cual se desarrolló esta investigación) se considera como una de las más longevas y con mayor porcentaje de adultos mayores en situación de pobreza (Encuesta de Caracterización Socieconomica [Casen], 2017).

Este envejecimiento a ritmo sostenido impone mayores exigencias a nivel sanitario, económico y psicosocial para los adultos mayores en Chile y América Latina (CEPAL, 2017; Ministerio de Desarrollo Social, 2012, OMS, 2015), representando desafíos tanto para quienes 
gozan de envejecimiento activo, como para quienes tienen discapacidades físicas y cognitivas severas y valorando que la vejez no es una etapa vital o patrón que determina a todos por igual (Mazzeti-Latini \& Crissi-Aloranti, 2018).

En las últimas décadas el concepto y la promoción del envejecimiento activo es relevante entre los temas de desarrollo socioeconómico mundial. Se entiende por envejecimiento activo "al proceso de optimización de las oportunidades de salud, participación y seguridad con el fin de mejorar la calidad de vida de las personas a medida que envejecen" (OMS, 2002, 79 p).

El interés por estudiar y promover el envejecimiento activo, también denominado envejecimiento saludable, "healthy ageing” (Mathers, Sadana, Salomon, Murray, \& Lopez, 2001), buen envejecimiento, "Ageing well" (Fries, 1980), envejecimiento exitoso, "successful ageing" (Minkler \& Fadem, 2002; Baltes, 1990) ó envejecimiento competente, “competent ageing” (Fernández-Ballesteros, Caprara, Iñiguez, \& García, 2005), es relativamente nuevo (ej. Castro omision Europea, 2012; Ferraea \& Zabala, 2014; García et al., 2010; OMS, 2002; Pugliese, 2014; Sánchez-valle, 2015). Esto debido a que la mayor parte de la investigación en ciencias de la salud, sociales y humanas se ha centrado en adultos mayores en condición de deterioro y/o discapacidad (e.j. Dreer, DeVivo, Novack, \& Marson, 2012; Fjell, McEvoy, Holland, Dale, \& Walhovd, 2014; Kolling et al., 2013; Krause, Jay, \& Liang, 1991; Lichtenberg, 2016; Minicuci, \& Bélanger, 2008; Pethtel \& Chen, 2010; Vega \& Rivera, 2003; Yoon, Hasher, Feinberg, Rahhal, \& Winocur, 2000).

La mayoría de intereses investigativos en la adultez mayor, reflejan la generalización de la imagen de las personas adultas mayores como enfermas, vulnerables y como una carga emotiva y económica para sus familias. Con la intención de cambiar estas ideas estereotipadas, la OMS (2002,2015), promueve la concepción y optimización del envejecimiento activo, para visibilizar el aporte que los adultos mayores hacen tanto a nivel familiar como social. En relación a lo anterior, en Chile, un estudio realizado por Adimark (2015) demostró que en el 30\% de los hogares del país, el principal sostenedor es un adulto mayor, lo que demuestra su rol activo a nivel socioeconómico.

$\mathrm{Al}$ respecto, en un estudio auspiciado por la Organización Panamericana de la Salud, el 66,7\% de los adultos mayores se autodefinió como capaces de llevar una vida independiente, 30\% como funcionalmente frágiles, es decir que requieren auxilio para ciertas actividades y sólo un $3.3 \%$ con invalidez severa y por tanto funcionalmente dependientes (Ministerio de Salud [Minsal], 2014), estos resultados coinciden con los de una encuesta nacional de dependencia en Chile 
(González et al., 2009). El Servicio Nacional del Consumidor [SERNAC] (2015), reconoce que los adultos mayores en Chile representan un segmento de consumidores creciente.

Si bien, las cifras anteriores son alentadoras, envejecer activamente releva asegurar y mejorar la calidad de vida de los adultos mayores y el pleno ejercicio de sus derechos como ciudadanos, lo que a su vez tiene implicaciones tanto físicas como psicosociales y por supuesto económicas. Por lo que las capacidades financieras tanto objetivas como percibidas, son un indicador importante, relacionado tanto con su calidad de vida como con el envejecimiento activo (OMS, 2015).

El concepto de calidad de vida ha ido evolucionando en los últimos años, desde una concepción centrada en los componentes objetivos del nivel de vida, hacia una perspectiva en la que el componente subjetivo constituye el elemento fundamental (Denegri, García \& González, 2015). Al componente subjetivo de la calidad de vida se le ha denominado bienestar subjetivo, el cual se compone de una dimensión afectiva, la felicidad y una dimensión cognitiva que es la satisfacción con la vida, ya sea de manera global o por dominios específicos (Cuadra, \& Florenzano, 2011; Moyano, Martínez, \& Muñoz, 2013; Moyano, Ramos, \& Alvarado, 2007; Pavot \& Diener, 2008; Schnettler et al., 2012). Si bien ha crecido el interés por los estudios en torno a la felicidad, en el presente trabajo se enfatiza en la dimensión de satisfacción con la vida, definida como un juicio global en el que el sujeto utiliza sus propios criterios para sopesar los distintos aspectos de su vida (Diener, Emmons, Larsen, \& Griffin, 1985).

Aunque se ha estudiado la satisfacción con la vida en la adultez mayor y en diferentes contextos culturales (Arraga \& Vera-Villarroel, 2010; Borg \& Blomqvist, 2006; Borg et al., 2008; Hernández, Fernández, Boto, \& Alonso, 2009; Whisman \& Judd, 2016; ), en Chile, la investigación al respecto es más limitada y reciente (Lobos \& Schnetler, 2015; Ramírez \& Lee Maturana, 2012) y no ha incluido otros dominios que resultan relevantes para determinar la posibilidad de autonomía en la adultez mayor (CEPAL, 2017), lo que se relaciona directamente con la calidad de vida en esta etapa de la vida.

Como se mencionó previamente, dentro de estos dominios, el estudio de las capacidades financieras resulta de particular interés desde la perspectiva del envejecimiento activo (Denegri, Sepulveda, Navarro \& Canching, 2018). Respecto a las capacidades financieras como dominio de la calidad de vida y su relación con esta, un antecedentes reciente y relevante, es un estudio realizado en la región Central en Chile, en el que se encontró que personas mayores están más 
satisfechas con su vida a medida que envejecen, cuando tienen mayor capacidad financiera y disponen de más dinero en sus bolsillos (Lobos \& Schnetler, 2015). Otro estudio reciente, asociado con las variables de interés, fue realizado en el sur de Chile, en el que se confirma la relación positiva y significativa entre el nivel de endeudamiento austero y la satisfacción con la vida (Denegri, Sepulveda, Navarro \& Canching, 2018).

Conceptualmente, las capacidades financieras incluyen el comportamiento, el conocimiento y la actitud de cada persona en relación a sus finanzas personales. Además implican la habilidad para manejar independientemente los asuntos financieros, de tal manera que sea consistente con los intereses propios y valores personales (Banco Mundial, 2013; García, Grifoni \& López, 2013; Gerstenecker et al., 2016; Lusardi, 2011; Lusardi, 2012; Marson et al., 2014; ).

De la misma manera que otros temas relacionados con la adultez mayor, las capacidades financieras sólo se han estudiado desde una perspectiva clínica y en el marco legal de la determinación de capacidades mentales o de interdicción para manejar sus posesiones y recursos financieros (Davies, Gilhooly, Gilhooly, Harries, \& Cairns, 2013; Flint, Sudore, \& Widera, 2012; Gerstenecker et al., 2016; Griffith et al., 2003; Lichtenberg, 2016; Marson et al., 2014; Stoeckel et al., 2013; Triebel \& Marson, 2012). Teniendo en cuenta la integración de los antecedentes hasta aquí planteados, el vacío y la relevancia tanto social, como teórica y empírica que implican, el objetivo de este estudio es explorar indicadores de satisfacción con la vida, dimensiones asociadas a las capacidades financieras y posibles relaciones entre estas variables en adultos mayores con envejecimiento activo en el sur de Chile.

El vacío teórico identificado, resulta relevante ante la realidad socioeconómica a la que se enfrentan los adultos mayores en Chile, considerando las bajas pensiones (Perinat Maceres \& Corral, 2001; OECD, BID \& World Bank, 2015) y los altos niveles de endeudamiento que reportan (SBIF, 2015). Según un estudio realizado por el Servicio Nacional del Consumidor [SERNAC] (2015), el 65\% de las personas mayores de 60 años, mantiene deudas en tarjetas de crédito de casas comerciales, y un $26 \%$ en créditos de consumo. Siendo además considerados como un segmento vulnerable respecto a temas de inclusión y alfabetización financiera (Denegri, et.al., 2018; García et al., 2013; Lusardi, 2012; Lusardi \& Mitchell, 2014; SBIF, 2015).

En términos específicos este estudio se orientó a: (a) Describir la satisfacción con la vida y dimensiones asociadas con las capacidades financieras de adultos mayores (b) Identificar las relaciones entre la satisfacción con la vida, seguridad e independencia en el manejo de las finanzas 
y actitudes hacia el endeudamiento en adultos mayores (c) Explorar diferencias por género, ocupación actual y etapa de la adultez mayor respecto a las capacidades financieras y satisfacción con la vida. Finalmente, como objetivo específico metodológico, se planteó (d) Explorar la consistencia interna y constitución factorial de las escalas de Satisfacción con la Vida, Independencia y Seguridad financiera y Actitudes hacia el Endeudamiento en adultos mayores.

\section{Método}

Diseño y Participantes

Estudio cuantitativo, con diseño exploratorio-descriptivo, de corte transversal (Hernández, 2010), con alcance correlacional. Se trabajó con una muestra intencionada no probabilística, constituida por 101 adultos mayores, que estuvo representada por mujeres en un 52\%, con un promedio de edad de 73,3 años $D E$ : 7,89; de los cuales el 55,4\% se encontraban en la etapa de adultez mayor temprana ó tercera edad (60-75 años) y 44,6\% de adultez mayor tardía ó cuarta edad (75 años en adelante), 29\% aun trabajan, distribuidos en los niveles socioeconómicos alto (13\%), medio (62\%) y bajo (25\%), principalmente casados $(67,3 \%)$ y viudos $(23,5 \%)$.

\section{Procedimiento}

Los participantes fueron invitados a participar voluntariamente del estudio, los instrumentos se aplicaron con asistencia de encuestadores entrenados y con previo acuerdo de consentimiento informado. Los cuestionarios fueron respondidos en organizaciones de adultos mayores, o bien en los lugares de trabajo o domicilio de los participantes. Los criterios de inclusión fueron: tener 60 años o más en el momento de la toma de datos, aceptar participar en el estudio y no tener deterioro cognitivo visible, es decir demostrar coherencia en la conversación, comprensión de instrucciones y ser capaces de responder preguntas simples.

\section{Instrumentos}

Escala de Satisfacción con la vida (Diener et al. 1985). En este estudio se empleó una versión de la escala traducida al español por Schnettler, Miranda, Sepúlveda, Denegri, Mora \& Lobos (2011), que contiene 5 ítems, con escala likert de 1-6 puntos, agrupados en un único factor. Con este instrumento se evalúan juicios cognitivos globales acerca de la propia vida (e.j. "En 
muchos aspectos mi vida se acerca a mi ideal"). La confiabilidad estimada con Alpha de Cronbach en varios estudios en países Latinoamericanos (Schnettler et al., 2014) ha oscilado entre 0,79 y 0,89. En Chile ha sido validada en población adulta mayor (Lobos \& Schnetler, 2015; Ramírez \& Lee, 2012) con consistencias internas entre 0,80-0,82, conservando su estructura unifactorial original.

Al tratarse de un estudio exploratorio y ante la ausencia de instrumentos específicos que midan las capacidades financieras en adultos mayores con envejecimiento activo, se seleccionaron las siguientes escalas que dan cuenta de dos de las dimensiones básicas de las capacidades financieras.

Escalas de Independencia y Seguridad Financiera. Utilizadas originalmente por Carpenter \& Moore (2008) en un estudio con universitarios. Por su parte, la Escala de Independencia Financiera, está compuesta por 3 ítems en formato likert de 1-5 (Totalmente en desacuerdo-Totalmente de acuerdo), hace referencia a la capacidad de ser responsables con la administración de las finanzas personales (e.j. Me describiría a mí mismo como financieramente independiente). El análisis de consistencia interna con Alpha de Cronbach de la escala en el estudio desarrollado por Carpenter \& Moore fue de 0,79 previo a la aplicación del instrumento, se eliminó un ítem (Estoy pagando mi educación universitaria), que no aplica a la realidad de los adultos mayores.

La Escala de Seguridad y Confianza Financiera, con 5 ítems, también en escala Likert de 1-5, alude a autopercibirse capaz del manejo de las finanzas personales y estar preparado para el futuro financiero (e.j. Tengo suficiente seguridad económica para afrontar gastos imprevistos de forma inmediata). El análisis de consistencia interna con Alpha de Cronbach de la escala en el estudio con población Universitaria fue de $0,86$.

Posterior al análisis factorial exploratorio (ver resultados) de ambas escalas, se depuraron dos ítems obteniendo una solución unifactorial de la escala que se ha denominado: Escala de Independencia y Seguridad Financiera.

Escala de Actitudes hacia el endeudamiento. La escala original está compuesta por 8 ítems en formato Likert de 4 puntos, que van desde totalmente en desacuerdo (1) a totalmente de acuerdo (4). Su objetivo es evaluar la actitud de las personas frente las deudas. Respecto a su validez factorial, se ha corroborado la presencia de 2 factores independientes: Hedonismo y Austeridad (Denegri, Estrada, \& Herrera, 2011; Denegri, Cabezas, Del Valle, González, 
Sepulveda, 2012; Mansilla, Denegri, \& Álvarez, 2016). En relación con las estimaciones de confiabilidad del instrumento, Araneda, Brahm \& García (2006) expresan que el factor «austeridad» presenta un nivel adecuado $(0,84)$, por su parte el factor «hedonismo» cuenta con un alfa aceptable $(0,68)$.

Cuestionario Sociodemográfico. Se le consultó a cada participante acerca de su género, edad, ocupación, nivel socioeconómico, estado civil y nivel educativo.

Análisis estadístico

Para analizar los datos se empleó el programa estadístico SPSS 24. Con el fin de dar cumplimiento al objetivo de explorar la consistencia interna y constitución factorial de las escalas de Satisfacción con la Vida, Independencia y Seguridad Financiera y Actitudes hacia el Endeudamiento, se ejecutó un análisis factorial exploratorio para cada una de las escalas, con análisis de componentes principales, considerando autovalores mayores que 1 y rotación de factores varimax (Pérez \& Medrano, 2010).

Conociendo el comportamiento de las escalas, se calculó la media y desviación estándar para cada una de las variables. Se realizó análisis exploratorio de puntuación total y por dimensiones de las escalas aplicadas. Para determinar diferencias significativas por género y etapa de la adultez mayor en la que se encontraban los participantes se realizaron pruebas t. El análisis de relación entre variables se efectuó con la prueba $r$ de Pearson.

\section{Resultados}

Exploración del comportamiento de las escalas

Previo probar los objetivos principales del estudio y para dar respuesta al objetivo metodológico de explorar la consistencia interna y constitución factorial de las escalas de Satisfacción con la Vida, Independencia, Confianza y Seguridad Financiera y Actitudes hacia el Endeudamiento en adultos mayores, se efectuó el análisis factorial exploratorio (AFE). Inicialmente, se reconoció el nivel de medición de las variables (Abad, Olea, Ponsoda, \& García, 2011) y con el coeficiente Alpha de Cronbach se probó la confiabilidad de las escalas, las cuales presentaron adecuados niveles de consistencia interna. 
Escala de Satisfacción con la Vida (SWLS). La escala de satisfacción con la vida, tuvo un Alpha de Cronbach de 0,85 y varianza explicada de 65\%, conservando la solución unifactorial original con 5 ítems (Tabla 1). Esta solución es similar a resultados obtenidos tanto en población con características similares, como en la validación de Diener et_al., (1985).

Tabla 1: Matriz de componentes rotados Escala de Satisfacción con la vida.

\begin{tabular}{|l|c|}
\hline \multicolumn{1}{|c|}{ Ítems } & $\begin{array}{c}\text { Factor: } \\
\text { Satisfacción con la vida }\end{array}$ \\
\hline En muchos aspectos su vida se acerca a su ideal & 0,758 \\
\hline Sus condiciones de vida son excelentes & 0,827 \\
\hline Está satisfecho con su vida & 0,815 \\
\hline $\begin{array}{l}\text { Hasta ahora ha obtenido las cosas importantes que ha } \\
\text { deseado en su vida }\end{array}$ & 0,768 \\
\hline $\begin{array}{l}\text { Si pudiera vivir su vida de nuevo, no cambiaría nada } \\
\text { Alpha de Cronbach }\end{array}$ & 0,806 \\
\hline Varianza Explicada & 0,650 \\
\hline \multicolumn{1}{|c|}{ Ala } & 0,851 \\
\hline
\end{tabular}

Elaboración Propia. Cargas factoriales para cada uno de los ítems de la escala original de satisfacción con la vida. Método de extracción: Análisis de componentes principales. Rotación: Varimax con Normalización de Kaisser.

Escala de Independencia y Seguridad Financiera. Del análisis factorial exploratorio (AFE), surge La reconfiguración de la escala con 5 ítems, la cual ha sido denominada de Independencia y Seguridad Financiera. Tuvo una consistencia interna con Alpha de Cronbach de 0,85, con varianza explicada del 63\% y solución unifactorial (Tabla 2). El resultado del AFE, sugiere suprimir dos ítems de la escala original (1. Soy responsable con mis finanzas y 2. He buscado ayuda financiera en otros).

Tabla 2: Matriz de componentes rotados Escala de Independencia y Seguridad Financiera

\begin{tabular}{|l|c|}
\hline \multicolumn{1}{|c|}{ Ítems } & \multicolumn{1}{|c|}{$\begin{array}{c}\text { Cargas factoriales } \\
\text { Factor: Independencia y } \\
\text { Seguridad Financiera }\end{array}$} \\
\hline $\begin{array}{l}\text { Me describiría a mí mismo como financieramente } \\
\text { independiente }\end{array}$ & 0,83 \\
\hline Siempre he manejado mis finanzas por mi cuenta & 0,81 \\
\hline $\begin{array}{l}\text { Confío en que puedo manejar mis finanzas sin ayuda de } \\
\text { otros }\end{array}$ & 0,80 \\
\hline Estoy bien preparado para enfrentar mi futuro financiero & 0,78 \\
\hline
\end{tabular}




\begin{tabular}{|l|c|}
\hline $\begin{array}{l}\text { Tengo suficiente seguridad económica para afrontar gastos } \\
\text { imprevistos de forma inmediata }\end{array}$ & 0,74 \\
\hline Varianza explicada & $63 \%$ \\
\hline Alpha de Cronbach & 0,85 \\
\hline
\end{tabular}

Elaboración Propia. Método de extracción: Análisis de componentes principales. Rotación: Varimax con Normalización de Kaisser.

Escala de actitudes hacia el endeudamiento. La Tabla 3 muestra los resultados del análisis factorial exploratorio de la escala de Actitudes hacia el Endeudamiento, que originalmente tiene 2 factores: Actitud Hedónica y Austera, los cuales se conservan. Fue necesario suprimir 2 ítems (1. El uso del crédito puede ser muy peligroso y 2. Es preferible tratar de pagar siempre al contado) con solución factorial compleja. Estas comunalidades fueron extraídas de los ítems mayor o igual a 0,5, carga factorial mayor o igual a 0,5 y carga cruzada inferior a 0,4, que además, de acuerdo con discusión con expertos, no daban cuenta de elementos contextuales de la realidad de los adultos mayores con quienes se hizo la exploración.

La consistencia interna, evaluada con Alpha de Cronbach, fue de 0,69 para actitud hedónica y 0,8 para actitud austera, con $36 \%$ y $26 \%$ de la varianza explicada respectivamente. Los resultados del análisis factorial exploratorio son consistentes con los obtenidos en otros estudios con población adulta chilena en cuanto a la distribución de los ítems para las sub escalas Austeridad y Hedonismo, aunque se conservaron 6 de 8 ítems originales.

Tabla 3: Matriz de componentes rotados Escala de Actitudes hacia el endeudamiento

\begin{tabular}{|l|c|c|} 
& \multicolumn{2}{|c|}{ Factores } \\
\hline Ítems & $\begin{array}{c}\text { Actitud } \\
\text { Austera }\end{array}$ & $\begin{array}{l}\text { Actitud } \\
\text { Hedónica }\end{array}$ \\
\hline Es importante pagar las deudas lo antes posible & 0,816 & \\
\hline Si uno se lo propone, siempre puede ahorrar algo de dinero & 0,807 & \\
\hline $\begin{array}{l}\text { La facilidad de obtener tarjetas de crédito es una causa del } \\
\text { endeudamiento de la gente }\end{array}$ & 0,781 & \\
\hline Hay que ser muy cuidadoso en el gasto del dinero & 0,756 & \\
\hline Es una buena idea comprar algo ahora y pagarlo después & & 0,850 \\
\hline Usar el crédito permite tener una mejor calidad de vida & & 0,782 \\
\hline Pedir un préstamo es a veces una muy buena idea & & 0,714 \\
\hline Varianza explicada (\%) & $36 \%$ & $26 \%$ \\
\hline Alpha de cronbach & 0,80 & 0,69 \\
\hline
\end{tabular}

Elaboración Propia. Método de extracción: Análisis de componentes principales. Rotación: Varimax con Normalización de Kaisser. 
Satisfacción con la Vida y Capacidades Financieras

En relación al cumplimiento del objetivo de describir indicadores de la satisfacción con la vida y dimensiones asociadas con las capacidades financieras de adultos mayores, se obtuvieron los siguientes resultados:

Satisfacción con la vida. Los resultados de este análisis exploratorio muestran que las personas adultas mayores del sur de Chile, se encuentran satisfechas con su vida, presentando mayor nivel de acuerdo respecto a que su vida en muchos aspectos se acerca a su ideal (70\%), si pudieran vivir su vida de nuevo no cambiarían nada (65\%), sus condiciones de vida son excelentes (64\%), están satisfechos con su vida (57\%) y hasta ahora han obtenido las cosas importantes que han deseado en su vida (56\%). El promedio de la escala de 1-6 puntos es de $\mathrm{M}=4,6=\mathrm{DE}=1,1$. La puntuación media total fue de 22,7 puntos, donde lo esperado para una mayor satisfacción con la vida de 30 puntos; esto indica que los adultos mayores participantes en el estudio, manifiestan un adecuado nivel de satisfacción con la vida. No se encontraron diferencias estadísticamente significativas por género, periodo de la adultez mayor en que se encuentran los participantes (tercera y cuarta edad) y entre personas que aun trabajan y quiénes no.

Capacidades financieras. Las variables asociadas con las capacidades financieras revelan que los participantes confían en que pueden manejar sus finanzas sin ayuda de otros (68\%), reconocen que siempre han manejado sus finanzas por su cuenta (62\%), manifiestan sentirse preparados para manejar su futuro financiero (62\%). De manera global, un 57\% se autoperciben como independientes financieramente y un $56 \%$ se sienten seguros para afrontar gastos e imprevistos de forma inmediata. El promedio obtenido en la escala de Independencia, Confianza y Seguridad financiera es de $\mathrm{M}=3,6 \mathrm{DE}=1,29$, con puntaje de 20 puntos, siendo el máximo esperado de 25 puntos. En cuanto a las actitudes hacia el endeudamiento, se encontró que los adultos mayores, muestran una actitud preponderantemente austera hacia el endeudamiento $(\mathrm{M}=$ 3,6 $\mathrm{DE}=0,72$ ), y una puntuación total de 14 puntos para un máximo de 16 , siendo mayor que la actitud hedónica $(\mathrm{M}=1,8 \mathrm{DE}=0,84)$, con 5,4 puntos para un máximo de 12 . No se encontraron diferencias estadísticamente significativas por género, periodo de la adultez mayor en que se encuentran los participantes (temprana y tardía) y entre personas que aun trabajan y quiénes no.

Exploración de relación entre variables. Se efectuó una correlación $r$ de Pearson para identificar posible relación entre las variables: Satisfacción con la Vida, Seguridad e Independencia Financiera y Actitudes hacia el endeudamiento en adultos mayores. Se encontró que existe relación 
positiva y significativa entre la satisfacción con la vida y la independencia y seguridad financiera en el manejo de las finanzas en adultos mayores $(r=0,367, p<0,001)$, así como con la actitud hacia el endeudamiento austera $(r=0,365, p<0,001)$, pero ninguna relación con la hedónica. Además se identifica relación entre la autopercepción de seguridad e independencia financiera y el estilo de endeudamiento austero $(r=0,356, p<0,001)$. No se identificaron diferencias significativas entre quienes se encuentran en la etapa de adultez mayor temprana (tercera edad) y tardía (cuarta edad), y tampoco con ninguna otra variable sociodemográfica.

\section{Discusión}

Algunas investigaciones sobre alfabetización e inclusión económica y financiera, asocian los déficits con determinados grupos sociodemográficos, tales como las mujeres, los jóvenes, adultos mayores, los grupos raciales pobres, los solteros y marginados (García et al., 2013; Lusardi \& Mitchell, 2009). Frente a la identificación de los adultos mayores como un grupo socioeconómico vulnerable ante la inclusión económica y financiera, los resultados obtenidos con esta primera investigación exploratoria, incentivan la profundización en posteriores estudios que desde la psicología económica, se interesen en comprender la participación de los mayores en el sistema económico y como esto impacta su bienestar ó viceversa.

Por otra parte, el crecimiento económico trae consigo la necesidad de que las personas sepan cómo manejar sus finanzas personales y beneficiarse de los mercados financieros más desarrollados. En este sentido, las iniciativas de educación financiera pueden convertirse en un complemento importante de los procesos de inclusión financiera y las medidas de reducción de la pobreza (García et al., 2013; Lusardi \& Mitchell, 2014). Atendiendo al objetivo de explorar tanto el comportamiento y ajuste de las escalas originales de Satisfacción con la Vida, Actitudes hacia el Endeudamiento, con sus sub escalas de Austeridad y Hedonismo, y la de Independencia y Seguridad financiera en población adulta mayor chilena, se encontró que se mantiene la configuración factorial original en la de Satisfacción con la vida, pero que fue necesario excluir algunos ítems en la escala de actitudes hacia el endeudamiento y la Independencia y Seguridad Financiera. Para esta última se definió una configuración unifactorial. No obstante, se recomienda para futuras investigaciones, explorar los resultados de ambas escalas con muestras más grandes, que permitan hacer un nuevo análisis factorial exploratorio y probar el modelo con un análisis factorial confirmatorio, con base en profundización en la revisión teórica. 
Como un aporte, surge la nueva versión unifactorial de la escala de Autopercepción de Independencia y Seguridad Financiera y la de Actitudes hacia el Endeudamiento ajustada para Adultos mayores, ambas con consistencia interna muy satisfactoria. A pesar de este aporte, surge la necesidad de desarrollar instrumentos que incluyan otras dimensiones de las capacidades financieras, tales como prácticas en la administración del dinero, planificación para eventos futuros, uso de productos y conocimientos financieros (Banco Mundial, 2013) en adultos mayores con envejecimiento activo en Chile, dado que el interés al respecto se ha centrado en la comprensión de la variable en adultos mayores con deterioro físico y/o mental (Marson et al., 2014; Gerstenecker et al., 2016).

Se deduce de estos resultados que el goce de la seguridad económica y la autopercepción de la misma, permite a las personas mayores satisfacer las necesidades objetivas que agregan calidad de vida a los años, y mantener la autoconfianza y nivel de independencia en la toma de decisiones. Todo ello refuerza su autovalencia al permitir la participación en la vida cotidiana como ciudadanos con plenos derechos (CEPAL, 2017, Huenchuan \& Guzmán, 2006; Perinat Maceres \& Corral, 2001).

En cuanto a las actitudes hacia el endeudamiento, los resultados indican la presencia de una actitud austera en los adultos mayores participantes del estudio. Esta tendencia actitudinal puede vincularse a la influencia de variables históricas y contextuales en el sur de Chile. Territorialmente, el sur de Chile ha sido una región de fuerte tradición agrícola y de latifundio, a pesar de los cambios en el desarrollo económico y financiero, esta tradición aun coexiste especialmente en las generaciones de mayor edad, quienes tienen fuertes tendencias culturales tradicionales ligadas a la valoración de la austeridad como forma de vida, la importancia del trabajo y el valor del esfuerzo y el sacrificio, así como un cierto rechazo a la ostentación y el lujo (Denegri, Sepúlveda, \& Godoy, 2011).

A lo anterior, se suma la presión permanente que experimentan para tomar decisiones complejas sobre la gestión de su dinero de modo que les permita preservar el frágil equilibrio entre los activos y los gastos en un momento de aumento de la longevidad y disminución de los ingresos (Denegri, et.al., 2018; Smith y Ekerdt, 2011).

El resultado anteriormente expuesto, difiere de lo encontrado por Denegri, Estrada, \& Herrera (2011), con personas en la etapa de la adultez media, trabajadores, quienes mostraron una actitud hacia el endeudamiento alta tanto en austeridad como en hedonismo, pero esto se explica si 
consideramos que son personas en etapa de desarrollo profesional, con mayores exigencias del mercado, competitividad y posible capacidad de endeudamiento para obtener bienes y servicios, lo que no ocurre con los adultos mayores. Por otro lado, los resultados de la investigación, demostraron que los adultos mayores presentan un adecuado nivel de satisfacción con la vida, esto acorde con otras investigaciones con las que se confirma que en la medida en que se avance en los niveles del desarrollo evolutivo se está más satisfecho con la vida (Arraga Barrios \& Villarroel, 2010; Denegri, et.al., 2018; Diener et al., 1985; Hernández et al., 2009; Lobos, \& Schnetler, 2015; Meléndez, Navarro, Oliver, \& Tomás, 2009; Palma-Candia, Ortega-Valdivieso, \& Cruz-Quintana, 2016.

Para futuras investigaciones se considera relevante la comparación de grupos con muestras más grandes y características sociodemográficas más homogéneas, dado que al tratarse de una primera exploración con un grupo pequeño, no se pueden identificar diferencias y hacer análisis de mayor potencia estadística. Los resultados de relaciones entre variables son consistentes con hallazgos en población adulta (Denegri, Estrada, \& Herrera, 2011; Denegri, Cabezas, Del Valle, González, Sepulveda, 2012; Mansilla et al., 2016), en la que se ha encontrado clara relación entre la austeridad con la satisfacción con la vida. Igualmente, a mayor independencia y seguridad financiera, mayor probabilidad de privilegiar valores austeros frente al endeudamiento. Así, los resultados de esta investigación aportan a pensar cómo enfrentar el envejecimiento de la población y su relación con la calidad de vida de los adultos mayores en Chile. Además, enfatizan en la necesidad de desarrollar instrumentos ajustados a las necesidades y características de los adultos mayores, que permitan efectivamente contribuir con resultados que orienten acciones dirigidas a mejorar su calidad de vida, educación, participación financiera y ejercicio pleno de derechos. Finalmente y a pesar de tratarse de un primer estudio exploratorio, los resultados proporcionan las bases para avanzar en el reconocimiento de características, patrones actitudinales y de comportamiento financiero de los adultos mayores y su relación con la satisfacción con la vida, considerados por la OMS, OPS, OCDE, SERNAC, SBIF y SENAMA, como un segmento de consumidores de interés tanto para la protección y promoción de su bienestar como para la educación, participación, evaluación y estimulación de sus capacidades financieras, como indicador de envejecimiento activo y calidad de vida (CEPAL, 2017; Estrada \& Ivonet, 2017; OMS, 2015). 


\section{Agradecimientos}

Núcleo Científico y Tecnológico de Ciencias Sociales y Centro de Excelencia en Psicología Económica (Universidad de la Frontera), Beca Conicyt CONICYT-PCHA/Doctorado Nacional/2015-21150294

\section{Referencias}

Abad, F., Olea, J., Ponsoda, V., \& García, C. (2011). Medición en ciencias Sociales y de la Salud. España: Síntesis.

Adimark, E. (2015). Adultos Mayores en Chile: Ojos puestos en el futuro. Adimark Chile, 1-31.

Arraga, M. V., \& Villarroel, M. (2010). Bienestar Subjetivo en Adultos Mayores Venezolanos. Interamerican Journal of Psychology, 44(1), 12-18.

Baltes, P., \& Baltes, M. (1990). Successful Aging: Perspectives from the behavioral sciencies. Cambridge: Cambridge University Press.

Banco Mundial. (2013). Capacidades financieras en Colombia. Banco Internacional de Reconstrucción y Fomento, 1-101.

Borg, C., Balducci, C., Burholt, V., Ferring, D., Weber, G., Wenger, C., \& Hallberg, I. R. (2008). Life Satisfaction in 6 European Countries: The Relationship to Health, Self-Esteem, and Social and Financial Resources among People (Aged 65-89) with Reduced Functional Capacity. Geriatric Nursing, 29(1).

Borg, C., \& Blomqvist, K. (2006). Life satisfaction among older people ( $65+$ ) with reduced self-care capacity: The relationship to social, health and financial aspects. Clinical Nursing, 15, 607-618.

Carpenter, J. M., \& Moore, M. (2008). Gender and credit behaviors among college students: Implications for consumer educators. Journal of Family \& Consumer Sciences Education, 26(1), 42-47.

Comisión Económica para América Latina y el Caribe. (2003). Estrategia Regional de Implementación para América Latina y El Caribe del Plan de Acción Internacional de Madrid. CEPAL, 1(1), 1-17.

Comisión Económica para América Latina y el Caribe. (2017). Derechos de las Personas Mayores. Retos para la Independencia y Autonomía. CEPAL, 1-173.

Comision Europea. (2012). La aportación de la UE al envejecimiento activo y a la solidaridad entre las generaciones. Comisión Europea, 20. https://doi.org/10.2767/67663

Cuadra L., H., \& Florenzano U., R. (2011). El Bienestar Subjetivo: Hacia una Psicología Positiva. Revista de Psicología, 12(1), Pág. 83-96. https://doi.org/10.5354/0719- 
0581.2003 .17380

Davies, M. L., Gilhooly, M. L. M., Gilhooly, K. J., Harries, P. A., \& Cairns, D. (2013). Factors influencing decision-making by social care and health sector professionals in cases of elder financial abuse. European Journal of Ageing, 10(4), 313-323. https://doi.org/10.1007/s10433-013-0279-3

Denegri, M., Cabezas, D., Del Valle, C., González, Y., \& Sepulveda, J. (2012). Escala de actitudes hacia el endeudamiento: Validez factorial y perfiles actitudinales en estudiantes universitarios chilenos. Universitas Psychologica, 11(2), 497-509. https://doi.org/16979267(201206)11:2<497:EAHEVF>2.0.TX;2-Y

Denegri, M; Estrada, C \& Herrera, M. (2011). La alfabetización económica, hábitos de consumo, actitud hacia el endeudamiento y su relación con el bienestar psicológico en funcionarios públicos de la ciudad de Punta Arenas. Magallania, 39(1), 83-92. https://doi.org/http://dx.doi.org/10.4067/S0718-22442011000100005

Denegri, M., Sepúlveda, J., \& Godoy, M. (2011). Actitudes hacia la compra y el consumo en estudiantes de pedagogia y profesores en ejercicio en chile. Psicología desde El Caribe, $28,1-23$.

Denegri, C., García, J., \& González, R. (2015). Definición de bienestar subjetivo en adultos jóvenes profesionales chilenos . Un estudio con redes. CES Psicología, 8(1), 77-97.

Denegri, M., Sepúlveda, J., Navarro, R.M., y Cánching, J. (2018). Bienestar subjetivo e inclusión financiera en adultos mayores social e intelectualmente activos en la Región de La Araucanía, Chile. En: Plaza, V (ed). Necesidades emergentes en Chile: propuestas para la promoción del envejecimiento activo desde la Psicología. Santiago: RiL Editores

Diener, E., Emmons, R., Larsen, R., \& Griffin, S. (1985). The Satisfaction With Life Scale. Journal of Personality Assessment. https://doi.org/10.1207/s15327752jpa4901_13

Dreer, L. E., DeVivo, M. J., Novack, T. a., \& Marson, D. C. (2012). Financial capacity following traumatic brain injury: A six-month longitudinal study. Rehabilitation Psychology, 57(1), 5-12. https://doi.org/10.1037/a0025818

Estrada, J., \& Ivonet, M. (2017). Seguridad económica de las pensiones mínimas de vejez en grupos etarios de adultos mayores en Cuba. Analysis, 20(7), 1-15. https://doi.org/10.5281/zenodo.1248223

Fernández-Ballesteros, R., Caprara, M., Iñiguez, J., \& García, L. (2005). Promoción del envejecimiento activo : Efectos del programa «Vivir con vitalidad» ${ }^{\circledR}$. Revista Española de Geriatría y Gerontología, 40(2), 92-103. https://doi.org/10.1016/S0211139X(05)74834-4

Ferraea, L., \& Zabala, M. (2014). Bienestar Psicológico: Adultos Mayores Activos a través del Voluntariado. Ciencia y Enfermería, 20(1), 123-130. https://doi.org/10.4067/S071795532014000100011 
Fjell, A. M., McEvoy, L., Holland, D., Dale, A. M., \& Walhovd, K. B. (2014). What is normal in normal aging? Effects of aging, amyloid and Alzheimer's disease on the cerebral cortex and the hippocampus. Progress in Neurobiology, 117, 20-40. https://doi.org/10.1016/j.pneurobio.2014.02.004

Flint, L., Sudore, R., \& Widera, E. (2012). Assessing Financial Capacity Impairment in Older Adults. Generations, 36(2), 59-65.

Fries, J. (1980). Aging, natural death, and the compression of morbidity. The New England Journal of Medicine, 130-135.

García, N., Grifoni, A., \& López, J. (2013). La educación financiera en América Latina y el Caribe. Situación actual y perspectivas. Banco de Desarrollo de América Latina (Vol. 12). Retrieved from http://www.oecd.org/daf/fin/financialeducation/OECD_CAF_Financial_Education_Latin_AmericaES.pdf

García, R. F., Dolores, M., Casinello, Z., Dolores, M., Bravo, L., Ángeles, M., ... Schettini, R. (2010). Envejecimiento con éxito : criterios y predictores. Psicothema, 22(4), 641-647.

Gerstenecker, A., Eakin, A., Triebel, K., Martin, R., Swenson-Dravis, D., Petersen, R. C., \& Marson, D. (2016). Age and education corrected older adult normative data for a short form version of the Financial Capacity Instrument. Psychological Assessment, 28(6), 737-749. https://doi.org/10.1037/pas0000159

Gerstorf, D., Ram, N., Goebel, J., Schupp, J., Lindenberger, U., \& Wagner, G. G. (2010). Where people live and die makes a difference: Individual and geographic disparities in wellbeing progression at the end of life. Psychology and Aging, 25(3), 661-676. https://doi.org/10.1037/a0019574

González, F., Massad, C., \& Lavanderos, F. (2009). Estudio Nacional de la dependencia en las Personas Mayores. SENAMA Gobierno de Chile, 10-12. Retrieved from http://www.senama.cl/filesapp/Estudio Nacional de Dependencia en las Personas Mayores.pdf

Griffith, H. R., Belue, K., Sicola, A., Krzywanski, S., Zamrini, E., Harrell, L., \& Marson, D. C. (2003). Impaired financial abilities in mild cognitive impairment: a direct assessment approach. Neurology, 60(3), 449-457. https://doi.org/10.1212/WNL.60.3.449

Hernández, C. R., Fernández, V. L., Boto, R. G., \& Alonso, T. O. (2009). Propiedades psicométricas de la escala de satisfacción con la vida en mujeres mayores activas. Revista Española de Geriatría y Gerontología, 44(3), 146-148. https://doi.org/10.1016/j.regg.2009.01.002

Hofer, J., Busch, H., Tavel, P., \& Wong, T. T. (2014). For the benefit of others: Generativity and meaning in life in the elderly in four cultures. Pychology and Aging, 29(4), 764-775.

Huenchuan, S. (2013). Envejecimiento, solidaridad y protección social en América Latina y el 
Caribe. La hora de avanzar hacia la igualdad. Naciones Unidas. CEPAL.

Huenchuan, S., \& Guzmán, M. (2006). Seguridad Económica y Pobreaza en la vejez: Tensiones, Expresiones y Desafíos para Políticas. Cepal, Santiago ((Fondo de Población de las Naciones Unidas), 1-23.

Instituto Nacional de Estadísticas (2012). Anuario de Estadísticas Vitales 2012. INE, Gobierno de Chile.

Instituto Nacional de Estadisticas (2017). Compendio Estadístico. INE, Gobierno de Chile.

Jang, Y., Roh, S., \& Chiriboga, D. (2014). The impact of acculturation on depressive symptoms: A comparison of older Korean Americans in two areas. Asian American Journal of Psychology, 5(3), 200-205. https://doi.org/10.1037/a0032591

Kim, J. E., Nesselroade, J. R., \& Featherman, D. L. (1996). The state component in self-reported worldviews and religious beliefs of older adults: the MacArthur Successful Aging Studies. Psychology and Aging, 11(3), 396-407. https://doi.org/10.1037/08827974.11.3.396

Kolling, T., Haberstroh, J., Kaspar, R., Pantel, J., Oswald, F., \& Knopf, M. (2013). Evidence and deployment-based research into care for the elderly using emotional robots. GeroPsych: The Journal of Gerontopsychology and Geriatric Psychiatry, 26(2), 83-88. https://doi.org/10.1024/1662-9647/a000084

Krause, N., Jay, G., \& Liang, J. (1991). Financial strain and psychological well-being among the American and Japanese elderly. Psychology and Aging, 6(2), 170-181. https://doi.org/10.1037/0882-7974.6.2.170

Smith, G. V. y Ekerdt, D. J. (2011). Confronting the material convoy in later life. Sociological Inquiry, 81(3), 377-391.

Lichtenberg, P. A. (2016). Financial exploitation, financial capacity, and Alzheimer 's disease. American Psychologist, 71(4), 312-320. https://doi.org/10.1037/a0040192

Lobos, G \& Schnetler, B. (2015). Indicadores de Bienestar subjetivo de Adultos Mayores del Maule. (Ivan Palomo, Ed.). Talca.

Lusardi, A \& Mitchell, O. S. (2009). Financial literacy: Evidence and implications for financial education. Trends and Issues, 1-18.

Lusardi, A. (2011). American financial capability. National bureau of economic research, Working Paper Series, 1(1), 1-26. https://doi.org/10.3386/w17103

Lusardi, B. A. (2012). Financial Literacy and Financial Decision-Making in Older Adults. Journal of the American Society on Aging, 36(2), 25-33. 
Lusardi, A., \& Mitchell, O. S. (2014). The Economic importance of financial literacy: Theory and Evidence. Journal of Economic Literature, 52(1), 5-44. https://doi.org/10.1257/jel.52.1.5

Mazzeti-Latini, C., \& Crissi-Aloranti, V. (2018). El envejecimiento poblacional como componente del ordenamiento territorial en la provincia de Córdoba, Argentina. Intersedes, 19(39), 43-61.

Mansilla, L., Denegri, M., \& Álvarez, B. (2016). Relación entre actitudes hacia el endeudamiento y locus de control del consumidor en estudiantes universitarios. Suma Psicológica, 23(1), 1-9. https://doi.org/10.1016/j.sumpsi.2015.11.002

Marson, D. C., Stalvey, T., Boothe, A., Aldridge, T., Chatterjee, A., \& Harrell, L. E. (2014). Assessing financial capacity in patients with alzheimer disease. Arch Neurol, 57(June 2000), 877-884.

Mathers, C., Sadana, R., Salomon, J., Murray, C., \& Lopez, A. (2001). World health report 2000 Healthy life expectancy in 191 countries, WHO, 357, 1685-1691.

Meléndez, J. C., Navarro, E., Oliver, A., \& Tomás, J. M. (2009). La satisfacción vital en los mayores. Factores sociodemográficos. Boletín de Psicología, (95), 29-42.

Minicuci, N., \& Bélanger, A. (2008a). Discapacidad y Esperanza de Vida Saludable : una comparación entre Italia , Bulgaria , Canadá , y siete ciudades de América Latina y el Caribe Asociación Latinoamericana de Población, 1-17.

Minkler, M., \& Fadem, P. (2002). “ Successful Aging :" Journal of Disability Policy Studies, 12(4), 229-235.

Ministerio de Desarrollo Social. (2012). Política integral de envejecimiento positivo para Chile 2012-2025. Servicio Nacional del Adulto Mayor. Gobierno de Chile, 1(1), 1-72.

Ministerio de Desarrollo Social. (2017). Encuesta Casen 2017: Región de La Araucanía: Caracterización de Adultos Mayores. Síntesis de resultados.

Ministerio de Salud (2014). Programa nacional de salud de las personas adultas mayores. Minsal, 239. https://doi.org/10.1017/CBO9781107415324.004

Moyano, E., \& Ramos, N. (2007). Bienestar subjetivo: Midiendo satisfacción vital, felicidad y salud en población Chilena de la región del Maule. Universum. Revista de Humanidades y Ciencias Sociales, 2(22), 184-200. https://doi.org/10.4067/S0718-23762007000200012

Moyano, E., Martínez, M., \& Muñoz, M. P. (2013). Propiedades psicométricas de la escala de satisfacción con la vida de Diener. Revista Argentina de Clínica Psicológica, 22 (1), $161-168$.

OECD, BID, \& World Bank. (2015). Panorama de las Pensiones: América Latina y El Caribe. Organización Mundial de la Salud. (2002). Envejecimiento activo: un marco político*. Rev Esp 
Geriat Gerontol, 37(52), 74-105.

Organización Mundial de la Salud. (2015). Informe Mundial sobre el Envejecimiento y la Salud. (1), 1-196. https://doi.org/10.1017/CBO9781107415324.004

Palma-Candia, O., Ortega-Valdivieso, A., \& Cruz-Quintana, F. (2016). Wellbeing of Chilean older adults is associated with group participation. Rev. Med Chile, 144, 1287-1296.

Pavot, W., \& Diener, E. (2008). The Satisfaction With Life Scale and the emerging construct of life satisfaction. The Journal of Positive Psychology, 3(2), 137-152. https://doi.org/10.1080/17439760701756946

Pérez, E \& Medrano, L. (2010). Análisis Factorial Exploratorio : Bases Conceptuales y Metodológicas. Artículo de Revisión. Revista Argentina de Ciencias Del Comportamiento, 2(1), 58-66.

Perinat Maceres, A., \& Corral Iñigo, A. (2001). Los adultos Mayores chilenos en el siglo XXI: un enfoque politológico. Acta Bioethica, 7(1), 71-95.

Pethtel, O., \& Chen, Y. (2010). Cross-cultural aging in cognitive and affective components of subjective well-being. Psychology and Aging, 25(3), 725-729.

Pugliese, L. (2014). El envejecimiento activo, aproximaciones conceptuales y ejemplos de programas que lo promueven. Asesoría General En Seguridad Social, 1(1), 67-91.

Ramírez, M., \& Lee, S. (2012). Factores asociados a la satisfacción de vida de adultos mayores de 60 años. Polis, 11(33), 407-427.

Sanhueza, M., Castro, M., \& Merino, J. M. (2005). Adultos mayores funcionales: un nuevo concepto en salud. Ciencia y Enfermería, 11(2), 17-21. https://doi.org/10.4067/S071795532005000200004

Schnettler, B., Miranda, H., Sepúlveda, J., Denegri, M., Mora, M., Lobos, G. (2011).

Satisfacción con la alimentación en personas Mapuches de la Región de La Araucania, Chile. Revista Chilena de Nutrición, 39(1), 18-29. https://doi.org/10.4067/S07175182012000100002

Schnettler Morales, B., Denegri, M., Miranda, H., Sepúlveda, J., Mora, M., \& Lobos, G. (2014). Satisfaction with life and with food-related life in central Chile. Psicothema, 26(2), 200206. https://doi.org/10.7334/psicothema2013.13

Servicio Nacional del Consumidor. (2015). Oferta de créditos de consumo para los adultos Stoeckel, L. E., Stewart, C. C., Griffith, H. R., Triebel, K., Okonkwo, O. C., Hollander, J. A., ... Marson, D. C. (2013). MRI volume of the medial frontal cortex predicts financial capacity in patients with mild Alzheimer's disease. Brain Imaging and Behavior, 7(3), 282-292. https://doi.org/10.1007/s11682-013-9226-3 
Superintendencia de Bancos e Instituciones Financieras. (2015). Informe de endeudamiento de los clientes bancarios. SBIF, 1-27.

Triebel, K. L., \& Marson, D. C. (2012). The warning signs of diminished financial capacity in Older Adults. Journal of American Society on Aging, 36(2), 39-46.

Vega, E., \& Rivera, C. (2003). Efecto agudo en los procesos cognitivos en adultos mayores al realizar ejercicio de fuerza a diferentes intensidades. Intersedes, 5(8), 1-

Whisman, M. A., \& Judd, C. M. (2016). A Cross-National Analysis of Measurement Invariance of the Satisfaction With Life Scale. Psychological Assessment, 28(2), 239-244. https://doi.org/10.1037/pas0000181

Yoon, C., Hasher, L., Feinberg, F., Rahhal, T. a., \& Winocur, G. (2000). Cross-Cultural Differences in Memory: The Role of Culture-BasedInStereotypes About Aging. Psychology and Aging, 15(4), 694-704. 